\title{
Tractable sufficient stability conditions for a system coupling linear transport and differential equations th
}

\author{
Mohammed Safi ${ }^{\mathrm{a}}$, Lucie Baudouin ${ }^{\mathrm{a}}$, Alexandre Seuret ${ }^{\mathrm{a}}$ \\ ${ }^{a} L A A S-C N R S$, Université de Toulouse, CNRS, Toulouse, France
}

\begin{abstract}
This paper deals with the stability analysis of a system of finite dimension coupled to a vectorial transport equation. We develop here a new method to study the stability of such a system, coupling ordinary and partial differential equations, using linear matrix inequalities led by the choice of an appropriate Lyapunov functional. To this end, we exploit Legendre polynomials and their properties, and use a Bessel inequality to measure the contribution of our approximation. The exponential stability of a wide class of delay systems is a direct consequence of this study, but above all, we are detailing here a new approach in the consideration of systems coupling infinite and finite dimensional dynamics. The coupling with a vectorial transport equation is a first step that already prove the interest of the method, bringing hierarchized conditions for stability. We will give exponential stability results and their proofs. Our approach will finally be tested on several academic examples.
\end{abstract}

Keywords: Transport equation, Lyapunov stability, Bessel inequality.

\section{Introduction}

Systems coupling partial and ordinary differential equations are one type of infinite dimensional systems. The robust control of what is also called distributed parameter systems has been a very active field for the last decades and has spawned several branches, such as e.g. in stability analysis and stabilization design. This article is meant to perform a stability analysis of a system of linear ordinary differential equations (ODE) coupled to a vectorial transport equation, which is a first order hyperbolic partial differential equation (PDE). Analysing and controlling this type of system coupling ODE

\footnotetext{
« The paper was partially supported by the ANR project SCIDIS. This paper was not presented at any IFAC meeting. Corresponding author. Fax +33-561336411.

Email address: msafi@laas.fr (Mohammed Safi)
} 
and PDE is an attractive topic at the interface of applied mathematics and automatic control. A large number of papers already exists on stability of this class of systems: see e.g. [20], [34], [28], [35], [26] among many others. For instance, such systems appear in the context of energy management as in [36]. The coupled system we will study in this article has the specificity to represent an alternative formulation of a time-delay system (TDS) with a vectorial transport equation replacing the delay terms. One should know that TDS also have a solution that evolves in an infinite dimensional space and in our opinion, this is an interesting connection that suggests to benefit of the different approaches for the stability and control in both domains (TDS and PDE). On the one hand, we can refer to several stability and control studies of PDE such as in the books $[2,22,9]$, and the references therein, or the nonexhaustive list of articles $[6,12,21]$ and [18]. On the other hand, TDS have been widely investigated in the literature (see e.g. [4, 15, 16, 17, 31], and [24]), and used in many areas, as in biological systems, mechanical transmissions or networked control systems.

One of the most fruitful fields of research in stability of these TDS relies on the exhibition of Lyapunov-Krasovskii functionals (LKF). In reference [16], the candidate Lyapunov functional called complete $L K F$, leads even to a necessary and sufficient stability condition. Nevertheless, the parameters composing this complete LKF make it numerically difficult to handle, especially for high dimensional systems $[14,23]$. A lot of investigations then turns to approximating these parameters, and more recently, approximation methods have been improved by considering polynomial like parameters of arbitrary degree [27].

Our goal in this article is to provide a novel framework to address stability problem of linear coupled finite/infinite dimensional systems following recent advances on time-delay systems presented for instance in [31]. Our work here is a first step towards more general PDE and focusses on the transport equation case, giving rise to a necesary stability criteria of stability when such a PDE is coupled to an ODE. The analysis adopted in the present paper is based on the Lyapunov theorem for infinite dimensional systems, which, according to us, represents a first relevant challenge. As a consequence of this more general analysis, we will provide a unified set of linear matrix inequalities (LMI) conditions allowed to guarantee exponential stability (in the sense of the $L^{2}$-norm) applicable to a wide class of delay systems including single/multiple/cross-talking delays for differential and difference equations, as particular case but is not only resume to these classes of systems. The objective of this paper is to provide a new framework for the analysis of this linear coupled ODE/hyperbolic PDE system. This contribution extends our preliminary studies presented in [3] and [29], where only a single transport speed was considered. The main difficulty is related to the infinite 
dimensional state of the system, which prevents from extending directly the existing methods of the finite dimension analysis. Nevertheless, in order to provide efficient and tractable stability conditions, we employ a polynomial approximation of the state expressed using Legendre polynomials, following the approach developed for TDS in [31].

Notations: $\mathbb{N}$ is the set of positive integer, $\mathbb{R}^{n}$ is the $n$-dimensional Euclidean space with vector norm $|\cdot|_{n} . I_{n}$ is the identity matrix in $\mathbb{R}^{n \times n}$, $0_{n, m}$ the null matrix $\in \mathbb{R}^{n \times m},\left[\begin{array}{cc}A & B \\ \star & C\end{array}\right]$ replaces the symmetric matrix $\left[\begin{array}{cc}A & B \\ B^{\top} & C\end{array}\right]$. We denote $\mathbb{S}^{n} \subset \mathbb{R}^{n \times n}$ (resp. $\mathbb{S}_{+}^{n}=\left\{P \in \mathbb{S}^{n}, P \succ 0\right\}$, and $\mathbb{D}_{+}^{n}$ ) the set of symmetric (resp. symmetric positive definite and diagonal positive definite) matrices and $\operatorname{diag}(A, B)$ is a bloc diagonal matrix. For any square matrix $A$, we define $\operatorname{He}(A)=A+A^{\top}$. Finally, $L^{2}\left(0,1 ; \mathbb{R}^{m}\right)$ represents the space of square integrable functions over the interval $[0,1] \subset \mathbb{R}$ with values in $\mathbb{R}^{m}$ and the partial derivative in time and space are denoted $\partial_{t}$ and $\partial_{x}$, while the classical derivative are $\dot{X}=\frac{d}{d t} X$ and $\mathcal{L}^{\prime}=\frac{d}{d x} \mathcal{L}$.

\section{Formulation of the problem}

\subsection{Linear coupled ODE-PDE system}

This article is devoted to the stability analysis of a system of ODEs coupled with a vectorial transport equation that takes the following shape:

$$
\begin{cases}\dot{X}(t)=A X(t)+B z(1, t), & t>0, \\ \partial_{t} z(x, t)+\Lambda \partial_{x} z(x, t)=0, & x \in(0,1), t>0 \\ z(0, t)=C_{1} X(t)+C_{2} z(1, t), & t>0, \\ z(x, 0)=z^{0}(x), & x \in(0,1) \\ X(0)=X^{0} & \end{cases}
$$

The state of this coupled system is composed of $X(t) \in \mathbb{R}^{n}$ and $z(\cdot, t) \in$ $L^{2}\left(0,1 ; \mathbb{R}^{m}\right)$. $A, B, C_{1}$ et $C_{2}$ are constant matrices with appropriate dimensions. The matrix of propagation speed $\Lambda \in \mathbb{D}_{+}^{m}$ is given by:

$$
\Lambda=\operatorname{diag}\left(\rho_{i} I_{m_{i}}\right)_{\{i=1 \ldots p\}} .
$$

Thus, each velocity $\rho_{i}>0$ is applied to $m_{i}$ components of the state $z(x, t)$ such that $m=\sum_{i=1}^{p} m_{i}$. Note that the situation of negative transport speed $\rho_{i}<0$, for some $i \leq p$ can be recast in the same formulation with positive transport speed using a change of state spatial variable $x^{\prime}=1-x$. The transport equation $\partial_{t} z+\Lambda \partial_{x} z=0$ in (1) of unknown $z=z(x, t)$ is a simple linear vectorial PDE and if the initial data $z^{0} \in L^{2}\left(0,1 ; \mathbb{R}^{m}\right)$ and the lateral boundary data $z(0, \cdot)=u \in L^{2}\left(\mathbb{R}_{+} ; \mathbb{R}^{m}\right)$ are given, it has a unique solution $z \in C\left(\mathbb{R}_{+} ; L^{2}\left(0,1 ; \mathbb{R}^{m}\right)\right)$ such that (see e.g. [9]), for all $t>0$ :

$$
\|z(t)\|_{L^{2}\left(0,1 ; \mathbb{R}^{m}\right)} \leq K\left(\left\|z^{0}\right\|_{L^{2}\left(0,1 ; \mathbb{R}^{m}\right)}+\|u\|_{L^{2}\left(\mathbb{R}_{+} ; \mathbb{R}^{m}\right)}\right) .
$$


Considering now the finite dimensional system in $X(t)$ coupled to the transport equation in the variable $z(x, t)$, we can notice that the coupling is linear and the existence of solution can be proved thanks to Theorem A.6 in [2]. Following this theorem, for every $z^{0} \in L^{2}\left(0,1 ; \mathbb{R}^{m}\right)$ and $X^{0} \in \mathbb{R}^{n}$, the Cauchy problem (1) has a unique solution. Moreover, there exist $K>0$ and $\delta>0$ such that the solution $(z(x, t), X(t))$ of system (1) satisfies :

$$
\|X(t)\|+\|z(t)\|_{L^{2}\left(0,1 ; \mathbb{R}^{m}\right)} \leq K e^{\delta t}
$$

This well-posedness result suggests the choice of the following total energy of the system $E(X(t), z(t))=|X(t)|_{n}^{2}+\|z(t)\|_{L^{2}\left(0,1 ; \mathbb{R}^{m}\right)}^{2}$, and in the sequel, we will denote $E(t)=E(X(t), z(t))$ in order to simplify the notations.

\subsection{Lyapunov stability}

We are looking for a candidate Lyapunov functional for (1) of the shape:

$$
\begin{array}{r}
V(X(t), z(t))=\int_{0}^{1} \int_{0}^{1}\left[\begin{array}{c}
X(t) \\
z\left(x_{1}, t\right)
\end{array}\right]^{\top}\left[\begin{array}{cc}
P & \mathcal{Q}\left(x_{1}\right) \\
\mathcal{Q}^{\top}\left(x_{2}\right) & \mathcal{T}\left(x_{1}, x_{2}\right)
\end{array}\right]\left[\begin{array}{c}
X(t) \\
z\left(x_{2}, t\right)
\end{array}\right] d x_{1} d x_{2} \\
+\int_{0}^{1} z^{\top}(x, t) e^{-2 \delta x \Lambda^{-1}}(S+(1-x) R) z(x, t) d x
\end{array}
$$

where the scalar $\delta>0$, the matrices $P \in \mathbb{S}_{+}^{n}, S, R \in \mathbb{S}_{+}^{m}$ and the functions $\mathcal{Q} \in L^{2}\left(0,1 ; \mathbb{R}^{n \times m}\right)$ and $\mathcal{T} \in L^{\infty}\left((0,1)^{2} ; \mathbb{S}^{m}\right)$ have to be determined. As for the energy, in the sequel, we will denote $V_{N}(t)=V_{N}(X(t), z(t))$ in order to simplify the notations.

Remark 1. Since the transport speed matrix belongs to $\mathbb{D}_{+}^{m}$, it is invertible and admits an inverse matrix $\Lambda^{-1}$ given by $\Lambda^{-1}=\operatorname{diag}\left(\rho_{i}^{-1} I_{m_{i}}\right)_{\{i=1 \ldots p\}}$. We can define its exponential matrix $e^{\Lambda^{-1}}=e^{\operatorname{diag}\left(\rho_{i}^{-1} I_{m_{i}}\right)}=\operatorname{diag}\left(e^{1 / \rho_{i}} I_{m_{i}}\right)$. This explains the term in $e^{-2 \delta x \Lambda^{-1}}$ in functional (3).

This functional is inspired by the complete Lyapunov-Krasovskii functional from [16] which is a necessary and sufficient condition for stability of linear systems with constant delay. It is however important to mention that the necessary conditions of [16] only states that if a linear time-delay systems is asymptotically stable, there exists parameters $P, Q, T, S$ and $R$ such that the associated functional is a Lyapunov-Krasovskii functional for the systems. That being said, the method developed in [16] may not be used to derive a tractable stability test for a given system.

Our functional $V$ defined in(3) is composed of four typical terms. The first quadratic term in $X(t)$ is dedicated to the state of the ODE, while the 
last three terms are concerned with the state $z(x, t)$ of the PDE. It is worth mentioning that the last two terms can be interpreted as the weighted energy of the transport equation (see for instance [9]) and have been widely used in the literature. The terms depending on $\mathcal{Q}$ are introduced to account for the coupling between the system of ODEs and the PDE. While this class of functionals is already classical for time delay systems, the interpretation of such functionals for PDEs is quite recent (see for instance [1] and [26]). The novelty of the present paper is closely related to these works. The difference of our approach relies on the use of efficient integral inequalities.

Our objectives in this paper are to provide an efficient numerical method to assess stability of system (1) by ensuring that the functional $V(X(t), z(t))$ in (3) is a Lyapunov functional for this system. As one will read in the proof of our stability result, it comes down to prove the existence of positive scalars $\varepsilon_{1}, \varepsilon_{2}$ and $\varepsilon_{3}$ such that the following inequalities hold

$$
\varepsilon_{1} E(t) \leq V(t) \leq \varepsilon_{2} E(t), \quad \dot{V}(t)+2 \delta V(t) \leq-\varepsilon_{3} E(t)
$$

The details will be developed in Section 4. Finally, we highlight that thanks to the general formulation of the coupled system (1) we can relate our work to stability studies of many types of time-delay systems. Among them, we can mention Systems with single or multiple constant delays which have been studied in many contribution on the subject (see [32], [19] and [7]) ; Systems with Cross-Talking delays (see [25]) ; Systems with commensurate (or, rationally dependent) delays, where a single delay and its multiples are involved(see e.g. [33]) ; Delay Difference systems, where the ODE is removed from the dynamics of (1) (see e.g. [11]). However, we insist that time-delay systems represent a particular subclass of systems generated by system (1).

\section{Main tools}

The objective of this section is to present our methodology to construct a candidate Lyapunov functional $V$ of shape (3). The ultimate objective is to provide tractable stability conditions for the infinite dimensional system, conditions that can be evaluated on numerical examples. While the parameters $P, S$ and $R$ are matrices, which can be easily defined and evaluated on numerical simulations, the main difficulty in the selection of a candidate functional relies on the definition of the functions $\mathcal{Q}$ and $\mathcal{T}$, that potentially vary with the integration parameters $x_{1}$ and $x_{2}$. Among the possible choices that can be found in the literature, we will focus on polynomial functions of a given degree, expressed using an orthogonal family of polynomials (e.g. the Legendre polynomials). This choice will be motivated in the sequel. 


\subsection{Construction of the Lyapunov functional}

The goal here is to provide a simple methodology to design the functions $\mathcal{Q}$ and $\mathcal{T}$ appearing in (3). To do so, we propose the following construction as a truncated decomposition of $\mathcal{Q}$ and $\mathcal{T}$ over specific polynomials:

$$
\mathcal{Q}(x)=\sum_{k=0}^{N} Q(k) \mathcal{L}_{k}(x), \quad \mathcal{T}\left(x_{1}, x_{2}\right)=\sum_{i=0}^{N} \sum_{j=0}^{N} T(i, j) \mathcal{L}_{i}\left(x_{1}\right) \mathcal{L}_{j}\left(x_{2}\right),
$$

where $N \in \mathbb{N}$, and where $\mathcal{L}_{k}$, for $k \in \mathbb{N}$, denote the shifted Legendre polynomials of degree $k$ considered over the interval $[0,1]$. More details about the definition of these polynomials and of their properties will be provided afterwards. Denoting $Q_{N}=[Q(i)]_{i=0 . . N}$ in $\mathbb{R}^{n, m(N+1)}, T_{N}=[T(i, j)]_{i, j=0 . . N}$ in $\mathbb{R}^{m(N+1), m(N+1)}$, and

$$
Z_{N}(t)=\left[\int_{0}^{1} z(x, t) \mathcal{L}_{0}(x) d x, \ldots, \int_{0}^{1} z(x, t) \mathcal{L}_{N}(x) d x\right]^{\top} \in \mathbb{R}^{m(N+1)},
$$

the Lyapunov functional (3) becomes easily $V_{N}(t)=V_{N, 1}(t)+V_{N, 2}(t)$ where

$$
\begin{aligned}
V_{N, 1}(t) & =\left[\begin{array}{c}
X(t) \\
Z_{N}(t)
\end{array}\right]^{\top}\left[\begin{array}{ll}
P & Q_{N} \\
* & T_{N}
\end{array}\right]\left[\begin{array}{c}
X(t) \\
Z_{N}(t)
\end{array}\right], \\
V_{N, 2}(t) & =\int_{0}^{1} z^{\top}(x, t) e^{-2 \delta x \Lambda^{-1}}(S+(1-x) R) z(x, t) d x .
\end{aligned}
$$

The interest of using (7) instead of (3) lies in the fact that the parameters defining $V_{N}$ are only matrices, and can be easily defined for numerical implementation. Nevertheless, this formulation requires an extensive studies of Legendre polynomials and the vector $Z_{N}(t)$, given in the next subsections. One should know that Lyapunov functional (7) is richer than those used in $[6,12,35]$. Indeed, the functionals considered in these papers represent a particular case of our general functional. In [6], matrices $Q, T$ and $R$ are not considered, while the result of $[12,35]$ can be recast as the particular case with $N=1$. The additional values of our approach consist than in taking into account the coupling between the ODE and the PDE through the projected state $Z_{N}$, for any arbitrary value of $N$.

\subsection{Shifted Legendre polynomials}

The shifted Legendre polynomials we will use are denoted $\left\{\mathcal{L}_{k}\right\}_{k \in \mathbb{N}}$ and act over $[0,1]$. It is crucial that the family $\left\{\mathcal{L}_{k}\right\}_{k \in \mathbb{N}}$ forms an orthogonal basis of $L^{2}(0,1 ; \mathbb{R})$ and we have precisely $\left\langle\mathcal{L}_{j}, \mathcal{L}_{k}>=\int_{0}^{1} \mathcal{L}_{j}(x) \mathcal{L}_{k}(x) d x=\frac{1}{2 k+1} \delta_{j k}\right.$, 
where $\delta_{j k}$ is Kronecker's coefficient, equal to 1 if $j=k$ and 0 otherwise. We denote the corresponding norm of this inner scalar product $\left\|\mathcal{L}_{k}\right\|=$ $\sqrt{\left\langle\mathcal{L}_{k}, \mathcal{L}_{k}\right\rangle}=1 / \sqrt{2 k+1}$. The polynomials also satisfy

$$
\mathcal{L}_{k}(0)=(-1)^{k}, \quad \mathcal{L}_{k}(1)=1, \quad \mathcal{L}_{k}^{\prime}(x)=\sum_{j=0}^{k-1} \ell_{k j} \mathcal{L}_{j}(x), \quad k \geq 1
$$

and, obviously $\mathcal{L}_{0}^{\prime}(x)=0$, with $\ell_{k j}= \begin{cases}(2 j+1)\left(1-(-1)^{k+j}\right), & \text { if } j \leq k-1, \\ 0, & \text { if } j \geq k .\end{cases}$ The definition of the polynomials and the proof of these properties are omitted here but can find for instance in [10].

\subsection{Truncated state}

Now, we have the tools to provide a study of the vector $Z_{N}(t)$ given in (6) and constructed for a prescribed integer $N$. We first note that the $m(N+1)$ components of $Z_{N}(t)$ are the projection of the $m$ components of the state $z(x, t)=\left(z_{i}(x, t)\right)_{i=1 \ldots m}$ of the transport equation over the $N+1$ first Legendre polynomials $\left\{\mathcal{L}_{k}\right\}_{k \in\{0 \ldots N\}}$ with respect to the canonical inner product of $L^{2}(0,1 ; \mathbb{R})$. One step towards the application of the Lyapunov theorem is the calculation of the time derivative of $Z_{N}(t)$. Let us first define some notations depending on $N$ :

$$
\begin{aligned}
& \mathbb{1}_{N}(\Lambda)=\left[\begin{array}{lll}
\Lambda & \ldots & \Lambda
\end{array}\right]^{\top}, \mathbb{1}_{N}^{*}(\Lambda)=\left[\begin{array}{llll}
\Lambda & -\Lambda & \ldots & (-1)^{N} \Lambda
\end{array}\right]^{\top} \in \mathbb{R}^{m(N+1), m} \\
& L_{N}(\Lambda)=\left[\begin{array}{ll}
\ell_{k j} \Lambda & ]_{j, k=0 . . N} \in \mathbb{R}^{m(N+1), m(N+1)}
\end{array}\right.
\end{aligned}
$$

where $\ell_{k j}$ is defined after (8) The following lemma provides an expression of the time derivative of this vector $Z_{N}(t)$, which will be useful in the sequel.

Lemma 1. Consider $z \in C\left(\mathbb{R}_{+} ; L^{2}\left(0,1 ; \mathbb{R}^{m}\right)\right)$ satisfying the transport equation in system (1). The time derivative of the vector $Z_{N}(t)$ is given by:

$$
\dot{Z}_{N}(t)=\left(\mathbb{1}_{N}^{*}(\Lambda) C_{2}-\mathbb{1}_{N}(\Lambda)\right) z(1, t)+\mathbb{1}_{N}^{*}(\Lambda) C_{1} X(t)+L_{N}(\Lambda) Z_{N}(t),
$$

using the notations defined in (9).

Proof : First, let us compute the time derivative of the projection of the infinite dimensional state $z(x, t)$ over the Legendre polynomial $\mathcal{L}_{k}$ for any $k$ 
in $\mathbb{N}$. Using the transport equation in (1), integration by parts and properties (8) of the Legendre polynomials, we can write:

$$
\begin{aligned}
& \frac{d}{d t} \int_{0}^{1} z(x, t) \mathcal{L}_{k}(x) d x=\int_{0}^{1} \partial_{t} z(x, t) \mathcal{L}_{k}(x) d x=-\int_{0}^{1} \Lambda \partial_{x} z(x, t) \mathcal{L}_{k}(x) d x \\
& =-\left[\Lambda z(x, t) \mathcal{L}_{k}(x)\right]_{0}^{1}+\int_{0}^{1} \Lambda z(x, t) \mathcal{L}_{k}^{\prime}(x) d x \\
& =-\Lambda z(1, t)+(-1)^{k} \Lambda z(0, t)+\sum_{j=0}^{\max [0, k-1]} \ell_{k j} \Lambda \int_{0}^{1} z(x, t) \mathcal{L}_{j}(x) d x .
\end{aligned}
$$

Consequently, using the notations recently introduced and omitting the time variable $t$, we have $\dot{Z}_{N}=-\mathbb{1}_{N}(\Lambda) z(1)+\mathbb{1}_{N}^{*}(\Lambda) z(0)+L_{N}(\Lambda) Z_{N}$. The proof is concluded by injecting the boundary condition $z(0, t)=C_{1} X(t)+$ $C_{2} z(1, t)$ in the previous expression.

\subsection{Bessel-Legendre inequality}

The following lemma gives a Bessel-type integral inequality that compares an $L^{2}(0,1)$ scalar product with the corresponding product of the finite dimensional approximations (built from the projection on the Legendre polynomials).

Lemma 2. Let $z \in L^{2}\left(0,1 ; \mathbb{R}^{m}\right)$ and $R \in \mathbb{S}_{+}^{m}$. Then the following inequality holds for all $N \in \mathbb{N}$

$$
\int_{0}^{1} z^{\top}(x) R z(x) d x \geq Z_{N}^{\top}\left[\begin{array}{lll}
R & & \\
& \ddots & \\
& & (2 N+1) R
\end{array}\right] Z_{N},
$$

Proof : It relies on the orthogonality of the Legendre polynomials and on the Bessel inequality, see e.g. [31]. More precisely, the proof of this lemma results easily from the expansion of $\int_{0}^{1} z_{N}^{\top}(x) R z_{N}(x) d x$, (and the fact it is positive definite) where $z_{N}(x)=z(x)-\sum_{k=0}^{N} \frac{\mathcal{L}_{k}(x)}{\left\|\mathcal{L}_{k}\right\|^{2}} \int_{0}^{1} z(y) \mathcal{L}_{k}(y) d y$ is the approximation error between the state $z$ and its projection $Z_{N}$ over the $N$ first Legendre polynomials .

Remark 2. Considering the Bessel-Legendre inequality with $N=0$ or 1 leads to the particular cases of the Jensen Inequality and the Wirtinger-based inequality [30]. Moreover, when $N$ tends to infinity, the inequality becomes an equality reflecting the well known Parseval identity. 
It is worth noting that $z_{N}$ is the unique polynomial that minimizes the distance between $z$ and the set of polynomials of degree less than $N$. This property results from the orthogonality of the Legendre polynomials. This also proves that inequality (11) is optimal. In addition, inequality (11) applied to $R=I_{m}$ can be interpreted as the $L^{2}$-norm of an element of $L^{2}\left(0,1 ; \mathbb{R}^{n}\right)$ being greater than the sum of the norm of its projections over the normalized version of the orthogonal sequence of Legendre polynomials. In that sense, this inequality refers indeed to the Bessel inequality on Hilbert spaces.

\section{Exponential stability result}

We provide here a stability result for System (1), whose proof is based on the proposed Lyapunov functional (7) and the use of Lemma 1 and Lemma 2. We first define the following set of matrices commuting with the transport speed matrix $\Lambda \in \mathbb{D}_{+}^{m}$ as follows

$$
\mathcal{M}_{\Lambda}^{m}:=\left\{M \in \mathbb{S}_{+}^{m}, M \Lambda=\Lambda M\right\} .
$$

Remark 3. $M \in \mathcal{M}_{\Lambda}^{m}$ if and only if $M \in \mathbb{S}_{+}^{m}$ is block diagonal and has the same Jordan structure as $\Lambda$ in $(2): M=\operatorname{diag}\left(M_{i}\right)_{i=1 \ldots p}$ with $M_{i} \in \mathbb{S}_{+}^{m_{i}}$.

We consider $S$ and $R$ in $\mathcal{M}_{\Lambda}^{m}$ and we define the following $\mathbb{R}_{+}^{m(N+1), m(N+1)}$ matrices on the model of $R^{N}$ in (2):

$$
\begin{aligned}
R^{N}(\Lambda) & =\operatorname{diag}\left(e^{-2 \delta \Lambda^{-1}} \Lambda R, 3 e^{-2 \delta \Lambda^{-1}} \Lambda R, \ldots,(2 N+1) e^{-2 \delta \Lambda^{-1}} \Lambda R\right), \\
S^{N}(\Lambda) & =\operatorname{diag}\left(e^{-2 \delta \Lambda^{-1}} S, 3 e^{-2 \delta \Lambda^{-1}} S, \ldots,(2 N+1) e^{-2 \delta \Lambda^{-1}} S\right), \\
\mathcal{I}^{N} & =\operatorname{diag}\left(I_{m}, 3 I_{m}, \ldots,(2 N+1) I_{m}\right) .
\end{aligned}
$$

Recall that the matrices $L_{N}(\Lambda), \mathbb{1}_{N}(\Lambda)$ and $\mathbb{1}_{N}^{*}(\Lambda)$ are defined in (9).

Theorem 1. Consider System (1) with a given transport speed $\Lambda \succ 0$. If there exists an integer $N>0$ such that there exists $\delta>0, P \in \mathbb{S}_{+}^{n}, Q_{N} \in$ $\mathbb{R}^{n,(N+1) m}, T_{N} \in \mathbb{S}^{(N+1) m}, S$ and $R \in \mathcal{M}_{\Lambda}^{m}$, satisfying the following LMIs

$\Phi_{N}(\Lambda, \delta)=\left[\begin{array}{cc}P & Q_{N} \\ * & T_{N}+S^{N}(\Lambda)\end{array}\right] \succ 0, \Psi_{N}(\Lambda, \delta)=\left[\begin{array}{ccc}\Psi_{11} & \Psi_{12} & \Psi_{13} \\ * & \Psi_{22} & \Psi_{23} \\ * & * & \Psi_{33}\end{array}\right] \prec 0$,

where

$$
\begin{aligned}
& \Psi_{11}=\operatorname{He}\left(P A+Q_{N} \mathbb{1}_{N}^{*}(\Lambda) C_{1}\right)+C_{1}^{\top} \Lambda(R+S) C_{1}+2 \delta P \\
& \Psi_{12}=P B+Q_{N}\left(\mathbb{1}_{N}^{*}(\Lambda) C_{2}-\mathbb{1}_{N}(\Lambda)\right)+C_{1}^{\top} \Lambda(R+S) C_{2}, \\
& \Psi_{13}=A^{\top} Q_{N}+C_{1}^{\top} \mathbb{1}_{N}^{* \top}(\Lambda) T_{N}+Q_{N} L_{N}(\Lambda)+2 \delta Q_{N}, \\
& \Psi_{22}=-e^{-2 \delta \Lambda^{-1}} \Lambda S+C_{2}^{\top} \Lambda(R+S) C_{2} \\
& \Psi_{23}=B^{\top} Q_{N}+\left(\mathbb{1}_{N}^{*}(\Lambda) C_{2}-\mathbb{1}_{N}(\Lambda)\right)^{\top} T_{N}, \\
& \Psi_{33}=\operatorname{He}\left(T_{N} L_{N}(\Lambda)\right)-R^{N}(\Lambda)+2 \delta T_{N},
\end{aligned}
$$


then, system (1) is exponentially stable. Moreover, for a given transport speed matrix $\Lambda \in \mathbb{D}_{+}^{m}$, there exists a constant $K>0$ and a guaranteed decay rate $\delta^{*}>\delta$ such that the energy of the system verifies, $\forall t>0$,

$$
E(t) \leq K e^{-2 \delta^{*} t}\left(\left|z^{0}(0)\right|_{m}^{2}+\left\|z^{0}\right\|_{L^{2}\left(0,1 ; \mathbb{R}^{m}\right)}^{2}\right) .
$$

Proof: Our objective is to show that the Lyapunov functional $V_{N}$ given in (7) verifies the inequalities

$$
\varepsilon_{1} E(t) \leq V_{N}(t) \leq \varepsilon_{2} E(t) \quad \text { and } \quad \dot{V}_{N}(t)+2 \delta V_{N}(t) \leq-\varepsilon_{3} E(t),
$$

for some positive scalars $\varepsilon_{1}, \varepsilon_{2}$ and $\varepsilon_{3}$. Therefore, the proof will successively relate the existence of each $\varepsilon_{i}$ to one of the LMI stated in Theorem 1 .

By proving that the Lyapunov functional verifies inequalities (16), we prove the exponential stability of system (1), since we get easily $\dot{V}_{N}(t)+$ $\left(2 \delta+\frac{\varepsilon_{3}}{\varepsilon_{2}}\right) V_{N}(t) \leq 0$. Integrating on the interval $(0, t)$ and using $2 \delta^{*}=2 \delta+\frac{\varepsilon_{3}}{\varepsilon_{2}}$, we obtain $V_{N}(t) \leq V_{N}(0) e^{-2 \delta^{*} t} \quad \forall t>0$. Using (16) once again, we get $\varepsilon_{1} E(t) \leq V_{N}(t) \leq V_{N}(0) e^{-2 \delta^{*} t} \leq \varepsilon_{2} E(0) e^{-2 \delta^{*} t}$, which corresponds to (15) that ends the proof of Theorem 1, provided that inequalities (16) are satisfied.

Existence of $\varepsilon_{1}$ : On the one hand, since $S \succ 0$ and $\Phi_{N} \succ 0$, there exists a sufficiently small $\varepsilon_{1}>0$ such that $\Phi_{N}=\left[\begin{array}{cc}P & Q_{N} \\ * & T_{N}+S^{N}(\Lambda)\end{array}\right] \succ \varepsilon_{1}\left[\begin{array}{cc}I_{n} & 0 \\ * & \mathcal{I}^{N}\end{array}\right]$ and $S \succ \varepsilon_{1} e^{2 \delta \Lambda^{-1}}$. On the other hand, $V_{N}$ defined by (7) satisfies, $\forall t \geq 0$,

$$
\begin{aligned}
V_{N}(t) \geq & {\left[\begin{array}{c}
X(t) \\
Z_{N}(t)
\end{array}\right]^{\top} \Phi_{N}(\Lambda, \delta)\left[\begin{array}{c}
X(t) \\
Z_{N}(t)
\end{array}\right] } \\
& +\int_{0}^{1} z^{\top}(x, t) e^{-2 \delta \Lambda^{-1}} S z(x, t) d x-Z_{N}^{\top}(t) S^{N}(\Lambda) Z_{N}(t) .
\end{aligned}
$$

Replacing $\Phi_{N}$ by its lower bound depending on $\varepsilon_{1}$ and introducing $\varepsilon_{1}$ in the last integral term, we have $V_{N}(t) \geq \varepsilon_{1}\left(|X(t)|_{n}^{2}+\int_{0}^{1} z^{\top}(x, t) z(x, t) d x\right)$ $+\int_{0}^{1} z^{\top}(x, t)\left(e^{-2 \delta \Lambda^{-1}} S-\varepsilon_{1} I_{m}\right) z(x, t) d x-Z_{N}^{\top}(t)\left(S^{N}(\Lambda)-\varepsilon_{1} \mathcal{I}^{N}\right) Z_{N}(t)$.

Since $S-\varepsilon_{1} e^{2 \delta \Lambda^{-1}} \succ 0$, applying Lemma 2 ensures that the expression on the second line is positive. We obtain a lower bound of $V_{N}(t)$ depending on the energy function $E(t): V_{N}(t) \geq \varepsilon_{1}\left(|X(t)|_{n}^{2}+\|z(t)\|_{L^{2}\left(0,1 ; \mathbb{R}^{m}\right)}^{2}\right) \geq \varepsilon_{1} E(t)$.

Existence of $\varepsilon_{2}$ : There exists a sufficiently large scalar $\beta>0$ that allows $\left[\begin{array}{cc}P & Q_{N} \\ Q_{N}^{\top} & T_{N}\end{array}\right] \preceq \beta\left[\begin{array}{cc}I_{n} & 0 \\ * & \mathcal{I}^{N}\end{array}\right]$, such that since $S \succ 0$ and $R \succ 0$, we get $V_{N}(t) \leq \beta|X(t)|_{n}^{2}+\beta Z_{N}^{\top}(t) \mathcal{I}^{N} Z_{N}(t)+\int_{0}^{1} z^{\top}(x, t) e^{-2 \delta x \Lambda^{-1}}(S+(1-x) R) z(x, t) d x$. 
Taking the upper bound of $(1-x)$ and the exponential term $e^{-2 \delta x \Lambda^{-1}}$ in $(0,1)$,

$$
V_{N}(t) \leq \beta|X(t)|_{n}^{2}+\beta Z_{N}^{\top}(t) \mathcal{I}^{N} Z_{N}(t)+\int_{0}^{1} z^{\top}(x, t)(S+R) z(x, t) d x .
$$

Applying Lemma 2 to the second therm of the right-hand side gives

$$
\begin{aligned}
V_{N}(t) & \leq \beta|X(t)|_{n}^{2}+\int_{0}^{1} z^{\top}(x, t)\left(\beta I_{m}+S+R\right) z(x, t) d x \\
& \leq \beta|X(t)|_{n}^{2}+\varepsilon_{2}\|z\|_{L^{2}\left(0,1 ; \mathbb{R}^{m}\right)}^{2} \leq \varepsilon_{2} E(t)
\end{aligned}
$$

where $\varepsilon_{2}=\beta+\lambda_{\max }(S)+\lambda_{\max }(R)$. Therefore, the proof of (16) is complete. Existence of $\varepsilon_{3}$ : We define here an augmented state vector, of size $n+$ $(N+2) m$ given by $\xi_{N}(t)=\left[\begin{array}{lll}X^{\top}(t) & z^{\top}(1, t) & Z_{N}^{\top}(t)\end{array}\right]^{\top}$. We can compute the derivative of $\left(X(t), Z_{N}(t)\right)$, using the first equation in system (1) and the new formulation of Lemma 1, omitting the variable $t$, as follows

$$
\frac{d}{d t}\left[\begin{array}{c}
X \\
Z_{N}
\end{array}\right]=\left[\begin{array}{c}
A \\
\mathbb{1}_{N}^{*}(\Lambda) C_{1}
\end{array}\right] X+\left[\begin{array}{c}
B \\
\mathbb{1}_{N}^{*}(\Lambda) C_{2}-\mathbb{1}_{N}(\Lambda)
\end{array}\right] z(1)+\left[\begin{array}{c}
0 \\
L_{N}(\Lambda)
\end{array}\right] Z_{N}
$$

Then, using (7), we can calculate $\dot{V}_{N, 1}$ :

$$
\dot{V}_{N, 1}=\frac{d}{d t}\left(\left[\begin{array}{c}
X \\
Z_{N}
\end{array}\right]^{\top}\left[\begin{array}{cc}
P & Q_{N} \\
Q_{N}^{\top} & T_{N}
\end{array}\right]\left[\begin{array}{c}
X \\
Z_{N}
\end{array}\right]\right)=\xi_{N}^{\top}\left[\begin{array}{ccc}
\psi_{1} & \psi_{2} & \psi_{3} \\
* & 0 & \Psi_{23} \\
* & * & \operatorname{He}\left(T_{N} L_{N}(\Lambda)\right)
\end{array}\right] \xi_{N},
$$

with $\psi_{1}=\operatorname{He}\left(P A+Q_{N} \mathbb{1}_{N}^{*}(\Lambda) C_{1}\right), \psi_{2}=P B+Q_{N}\left(\mathbb{1}_{N}^{*}(\Lambda) C_{2}-\mathbb{1}_{N}(\Lambda)\right)$, and $\psi_{3}=A^{\top} Q_{N}+C_{1}^{\top} \mathbb{1}_{N}^{* \top}(\Lambda) T_{N}+Q_{N} L_{N}(\Lambda)$, and $\Psi_{23}$ is already given in Theorem 1. On the other hand, one can write, using the transport equation in (1) and since $S$ and $R \in \mathcal{M}_{\Lambda}^{m}$,

$$
\begin{aligned}
& \dot{V}_{N, 2}=-\int_{0}^{1} \partial_{x}\left(z^{\top}(x) e^{-2 \delta x \Lambda^{-1}} \Lambda(S+(1-x) R) z(x)\right) d x \\
& -2 \delta \int_{0}^{1} z^{\top}(x) e^{-2 \delta x \Lambda^{-1}}(S+(1-x) R) z(x) d x-\int_{0}^{1} z^{\top}(x) e^{-2 \delta x \Lambda^{-1}} \Lambda R z(x) d x \\
& =\left[-z^{\top}(x) e^{-2 \delta x \Lambda^{-1}}(\Lambda(S+(1-x) R)) z(x)\right]_{0}^{1} \\
& -2 \delta V_{N, 2}-\int_{0}^{1} z^{\top}(x) e^{-2 \delta x \Lambda^{-1}} \Lambda R z(x) d x .
\end{aligned}
$$

From the boundary condition $z(0, t)=C_{1} X(t)+C_{2} z(1, t)$, we get

$$
\begin{aligned}
\dot{V}_{N, 2}(t)= & \left(C_{1} X+C_{2} z(1)\right)^{\top} \Lambda(S+R)\left(C_{1} X+C_{2} z(1)\right) \\
& -z(1)^{\top} e^{-2 \delta \Lambda^{-1}} \Lambda S z(1)-2 \delta V_{N, 2}-\int_{0}^{1} z^{\top}(x) e^{-2 \delta x \Lambda^{-1}} \Lambda R z(x) d x .
\end{aligned}
$$


Merging the expressions of $\dot{V}_{N, 1}$ and $\dot{V}_{N, 2}$ and using the definition of the matrix $\Psi_{N}(\Lambda, \delta)$ in (14) and $R^{N}$ in (13), we obtain $\dot{V}_{N}(t)+2 \delta V_{N}(t)=$ $\xi_{N}^{\top}(t) \Psi_{N}(\Lambda, \delta) \xi_{N}(t)-\int_{0}^{1} z^{\top}(x, t) e^{-2 \delta x \Lambda^{-1}} \Lambda R z(x, t) d x+Z_{N}^{\top}(t) R^{N}(\Lambda) Z_{N}(t)$. Taking the lower bound of the exponential in the integral term yields

$$
\begin{aligned}
\dot{V}_{N}(t)+2 \delta V_{N}(t) & \leq \xi_{N}^{\top}(t) \Psi_{N}(\Lambda, \delta) \xi_{N}(t) \\
& -\int_{0}^{1} z^{\top}(x, t) e^{-2 \delta \Lambda^{-1}} \Lambda R z(x, t) d x+Z_{N}^{\top}(t) R^{N}(\Lambda) Z_{N}(t),
\end{aligned}
$$

and applying Lemma 2 to the resulting term, the following inequality holds $\dot{V}_{N}(t)+2 \delta V_{N}(t) \leq \xi_{N}^{\top}(t) \Psi_{N}(\Lambda, \delta) \xi_{N}(t)$. Thereafter, the second LMI in (14) ensures the existence of $\varepsilon_{3}$ sufficiently small such that $\Psi_{N}(\Lambda, \delta) \prec-\varepsilon_{3}\left[\begin{array}{ccc}I_{n} & 0 & 0 \\ * & 0 & 0 \\ * & * & \mathcal{I}^{N}\end{array}\right]$ and we have also, for the same scalar $\varepsilon_{3}, \Lambda R \succ \varepsilon_{3} e^{2 \delta \Lambda^{-1}}$. Hence, using these two matrix inequalities in estimate (18), one obtains $\dot{V}_{N}(t)+2 \delta V_{N}(t) \leq$ $-\int_{0}^{1} z^{\top}(x, t)\left(e^{-2 \delta \Lambda^{-1}} \Lambda R-\varepsilon_{3} I_{m}\right) z(x, t) d x+Z_{N}^{\top}(t)\left[R^{N}(\Lambda)-\varepsilon_{3} \mathcal{I}^{N}\right] Z_{N}(t)-$ $\varepsilon_{3}|X(t)|_{n}^{2}-\varepsilon_{3} \int_{0}^{1}|z(x, t)|^{2} d x$. Since $e^{-2 \delta \Lambda^{-1}} \Lambda R-\varepsilon_{3} I_{m} \succ 0$, Lemma 2 can be applied and ensures that the expression in the second line of the previous equation is positive, so that the Lyapunov functional $V_{N}$ satisfies (16). One can therefore conclude on the exponential stability of system (1) with respect to the norm $E(t)$.

Remark 4. The general results proved here include the particular case presented in [3] that involves only one transport speed $\left(\Lambda=\rho I_{m}\right)$ for all the components of the transport variable $z(x, t)$.

\section{Hierarchy of LMI conditions}

Following the previous studies on delay systems with Bessel-Legendre inequality (e.g. [31]), the stability conditions of Theorem 1 form a hierarchy of LMI conditions. This is formulated in the following theorem.

Theorem 2. Considering the coupled system (1), and using the notations introduced for Theorem 1, define, for a given $\delta>0$, the set $\mathcal{P}_{N}(\delta) \subset \mathbb{D}_{+}^{m}$ by

$$
\mathcal{P}_{N}(\delta):=\left\{\begin{array}{c}
\Lambda \in \mathbb{D}_{+}^{m} \text { such that } \Phi_{N}(\Lambda, \delta) \succ 0, \Psi_{N}(\Lambda, \delta) \prec 0, \\
\text { for } P \in \mathbb{S}_{+}^{n}, S, R \in \mathcal{M}_{\Lambda}^{m}, T_{N} \in \mathbb{S}^{(N+1) m}, Q_{N} \in \mathbb{R}^{n,(N+1) m}
\end{array}\right\} .
$$

Thus, for all $\left(N, N^{\prime}\right) \in \mathbb{N}^{2}$ and all $\delta \in \mathbb{R}, N<N^{\prime} \Rightarrow \mathcal{P}_{N}(\delta) \subset \mathcal{P}_{N^{\prime}}(\delta)$. 
The set $\mathcal{P}_{N}(\delta)$, for a given positive scalar $\delta$, represent the set of transport speed matrices $\Lambda$ that are proven to give an exponentially stable system (1) with decay rate $\delta$, according to the conditions of Theorem 1 at the order $N$. The inclusion stated here means that increasing $N$ in the conditions of Theorem 1 can only enlarge the set of allowable speed matrices $\Lambda$.

Proof : Let us consider a given $\delta>0$ and two integers $N<N^{\prime}$. Without loss of generality, assume that $N^{\prime}=N+1$. If $\mathcal{P}_{N}(\delta)$ is empty, the inclusion is easily obtained. If $\mathcal{P}_{N}(\delta)$ is not empty, then for a given transport speed matrix $\Lambda \in \mathcal{P}_{N}(\delta)$, we have from the definition of $\mathcal{P}_{N}(\delta)$ the LMIs $\Phi_{N}(\Lambda, \delta) \succ 0$ and $\Psi_{N}(\Lambda, \delta) \prec 0$ satisfied for $\delta \geq 0, P \in \mathbb{S}_{+}^{n}, T_{N} \in$ $\mathbb{S}_{+}^{(N+1) m}, Q_{N} \in \mathbb{R}_{+}^{n,(N+1) m}, S$ and $R \in \mathcal{M}_{\Lambda}^{m}$. Selecting the matrices

$$
Q_{N+1}=\left[\begin{array}{ll}
Q_{N} & 0_{n, m}
\end{array}\right] \in \mathbb{R}^{n, m(N+2)}, T_{N+1}=\left[\begin{array}{cc}
T_{N} & 0_{m, m(N+1)} \\
* & 0_{m, m}
\end{array}\right] \in \mathbb{R}^{m(N+2), m(N+2)},
$$

and keeping $S$ and $R$, let us express the matrices $\Phi_{N+1}(\Lambda, \delta)$ and $\Psi_{N+1}(\Lambda, \delta)$ for this specific selection. The matrix $\Phi_{N+1}(\Lambda, \delta)$ can be written as $\Phi_{N+1}(\Lambda, \delta)$ $=\left[\begin{array}{cc}\Phi_{N}(\Lambda, \delta) & 0_{n+m(N+1), m} \\ * & e^{-2 \delta \Lambda^{-1}}(2 N+3) S\end{array}\right]$. Since $S \in \mathcal{M}_{\Lambda}^{m} \subset \mathbb{S}_{+}^{m}$ and $\Phi_{N}(\Lambda, \delta) \succ 0$ by assumption, then $\Phi_{N+1}(\Lambda, \delta) \succ 0$ also holds. For the remaining LMI to be proven, we note that the matrices $L_{N+1}(\Lambda)$ and $R^{N+1}(\Lambda)$ can be written as

$L_{N+1}=\left[\begin{array}{cc}L_{N}(\Lambda) & 0_{m(N+1), m} \\ {\left[\ell_{N+1,0} \Lambda, \ldots, \ell_{N+1, N} \Lambda\right]} & 0_{m, m}\end{array}\right], R^{N+1}=\left[\begin{array}{cc}R^{N}(\Lambda) & 0_{m(N+1), m} \\ * & (2 N+3) e^{-2 \delta \Lambda^{-1}} \Lambda R\end{array}\right]$.

From these expressions, the matrix $\Psi_{N+1}(\Lambda, \delta)$ depending on $L_{N+1}(\Lambda)$ and $R^{N+1}(\Lambda)$ can also be written as $\Psi_{N+1}(\Lambda, \delta)=\left[\begin{array}{cc}\Psi_{N}(\Lambda, \delta) & 0_{n+m(N+2), m} \\ * & -(2 N+3) e^{-2 \delta \Lambda^{-1}} \Lambda R\end{array}\right]$. Since $\Psi_{N}(\Lambda, \delta) \prec 0, \Lambda \in \mathbb{D}_{+}^{m}$ and $R \in \mathbb{S}_{+}^{m}$, we get $\Psi_{N+1}(\Lambda, \delta) \prec 0$, and we conclude that $\mathcal{P}_{N}(\delta) \subset \mathcal{P}_{N+1}(\delta)$. Finally, for any $N^{\prime}>N$, the inclusion $\mathcal{P}_{N}(\delta) \subset \mathcal{P}_{N^{\prime}}(\delta)$ is obtained by a recursive reasoning.

\section{Numerical examples}

This section aims at illustrating our approach by several examples taken from the literature. The first example borrowed from [6, 35] describes the dynamics of an experimental setup. The three last examples can be seen as academic ones mainly presented to evaluate the potential and accuracy of our method.

\subsection{Example 1}

In this example we consider the experimental setup studied in $[8,13$, 35 ] treating the stability of a gas flow transport model. It consists of two 


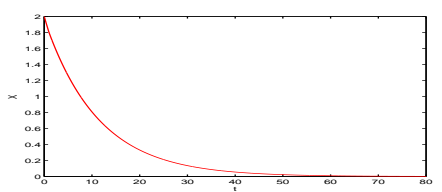

(a) Evolution of the ODE state $X$ depends on time.

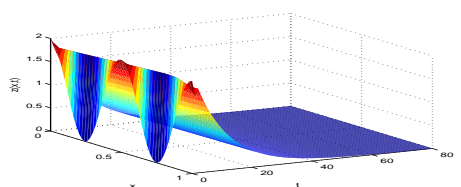

(b) Evolution of the PDE state $z$ depends on time $t$ and space $x$.

Figure 1: Evolution of different states in Examples 1.

subsystems: the heating column and the tube. The gas dynamic in the heating column is modeled by $\dot{\tau}(t)=-\frac{R \gamma T_{i n} \dot{m}_{i n}}{p_{i n} V_{0}} \tau(t)-\frac{R}{p_{i n} V_{0} C_{v}} \tau(t) d Q+\frac{\gamma \dot{m}_{i n}}{V_{0}}$, (see [5] and [35]), where $\tau$ is the gas density in the heating column, $R$ is the specific gas constant, $T_{i n}$ is the gas temperature at the input. $V_{0}$ represents the volume of the heating column and $\dot{m}_{i n}$ is the mass flow. The parameter $d Q$ is the heating exchange and $C_{v}$ is the specific heat of a constant volume of gas. On the other hand, the gas dynamic in the tube is described by a simple transport equation $z_{t}(x, t)+\rho z_{x}(x, t)=0$. Following the same procedure as [35], we consider the linear system under the form of system (1) with $A=-0.1, B=0.01, C_{1}=1, C_{2}=0$ and the velocity $\rho=1$. Solving the stability conditions presented in Theorem 1 , this system is proven to be exponentially stable with the exponential decay rate $\delta=0.089$.

In order to illustrate this example, the solution of system (1) with these matrices and with the initial conditions $X(0)=2$ and $z(x, 0)=\cos (4 \pi x)+1$, is drawn in Figure $1(\mathrm{a}, \mathrm{b})$, which is consistent with Theorem 1.

\subsection{Example 2}

Consider system (1) with following matrices

$$
A=\left[\begin{array}{cc}
0 & 1 \\
-2 & 0.1
\end{array}\right], B=\left[\begin{array}{cc}
0 & 0 \\
1 & 0.1
\end{array}\right], C_{1}=\left[\begin{array}{ll}
1 & 0 \\
0 & 0
\end{array}\right], C_{2}=\left[\begin{array}{cc}
0 & 0 \\
1 & 0.5
\end{array}\right], \Lambda=\rho I_{2}
$$

We first note that matrix $A+B C_{1}=\left[\begin{array}{cc}0 & 1 \\ -1 & 0.1\end{array}\right]$ is not Hurwitz. Therefore, it means that this system is not asymptotically stable for an arbitrary large transport speed $\rho$. The requirement imposing the matrix $A+B C_{1}$ to be Hurwitz is required in $[6,35]$. This is however not a necessary condition for the resulting ODE/PDE system to be asymptotically stable. The following Table 1 demonstrates that Theorem 1 is still able to guarantee exponential stability even if $A+B C_{1}$ is not assumed to be Hurwitz. In this situation, it appears that the stability regions are bounded interval and more especially, there exist minimal and maximal values of $\rho$ such that the overall system (1) remains exponentially stable. Table 1 also depicts the evolution of these 


\begin{tabular}{|c|c|c|c|c|c|}
\hline & & $\mathrm{N}=0$ & $\mathrm{~N}=1$ & $\mathrm{~N}=2$ & $\mathrm{~N}=3$ \\
\hline Variables & & 8 & 12 & 17 & 23 \\
\hline$\delta=0$ & $\rho_{\text {min }}$ & - & 0.653 & 0.582 & 0.579 \\
& $\rho_{\text {max }}$ & - & 15.924 & 15.941 & 15.941 \\
\hline$\delta=5.10^{-3}$ & $\rho_{\min }$ & - & 0.661 & 0.587 & 0.584 \\
& $\rho_{\max }$ & - & 14.470 & 14.490 & 14.490 \\
\hline$\delta=1.10^{-2}$ & $\rho_{\min }$ & - & 0.670 & 0.593 & 0.589 \\
& $\rho_{\max }$ & - & 13.259 & 13.282 & 13.282 \\
\hline$\delta=5.10^{-2}$ & $\rho_{\min }$ & - & 0.738 & 0.634 & 0.625 \\
& $\rho_{\max }$ & - & 7.935 & 7.980 & 7.980 \\
\hline
\end{tabular}

Table 1: Minimal and maximal allowable transport speed in example 1.

minimal and maximal values of $\rho$ for several values of $\delta$ and $N$. One can see from this table that increasing $N$ allows enlarging the length of the interval $\left[\rho_{\min }, \rho_{\max }\right]$, which illustrates the hierarchical structure of the LMI with respect to degree of Legendre polynomials $N$ Theorem 2 . On the other hand,we can note from this values that increasing $\delta$ obviously reduces the length.

\subsection{Example 3}

In this example, we deal with a system with multiple transport speeds, specifically with $\Lambda=\operatorname{diag}\left(\rho_{1}, \rho_{2}\right)$. Let us consider system 1 with matrices

$$
A=[-1.3], B=\left[\begin{array}{ll}
-1 & -0.5
\end{array}\right], C_{1}=\left[\begin{array}{l}
1 \\
1
\end{array}\right], C_{2}=\left[\begin{array}{ll}
0 & 0 \\
0 & 0
\end{array}\right] .
$$

The stability regions for different value of $\rho_{1}$ and $\rho_{2}$ are given by Figure 2(a). We can notice that increasing $N$ allows us to broaden the stability region of the coupled system. Figure 2 (a) also illustrates the principles presented in Theorem 2 since we can see that $\mathcal{P}_{1} \subset \mathcal{P}_{3} \subset \ldots \subset \mathcal{P}_{9}$.

In order to evaluate the conservatism of our approach, we benefits from the fact that this system has been already studied in the context of timedelay systems in [32]. Indeed, this systems can be rewritten as $\dot{X}(t)=$ $-1.3 X(t)-X\left(t-\rho_{1}^{-1}\right)-0.5 X\left(t-\rho_{2}^{-1}\right)$. The stability regions calculated using a frequency domain approach as in [32] are including in Figure 2(a), where it can be seen that the stability region we derive here for $N=9$ provides a very inner approximation of the exact stability regions. It is true that Theorem 1 is only able to provide an inner approximation of the stability regions, which can be calculated in an exact manner using a frequency approach. However, the frequency approach does not ensure robustness, which corresponds to the main advantage of our Lyapunov approach. 


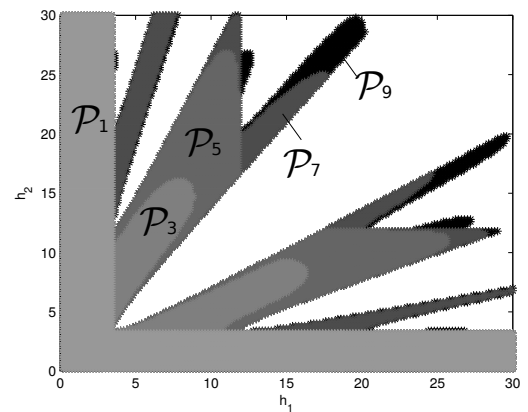

(a) Figure representing the unstable region (white area) and the stability regions guaranteed by Theorem 1 with $\delta=0$ and for several values of $N$ from 1 to 9 .

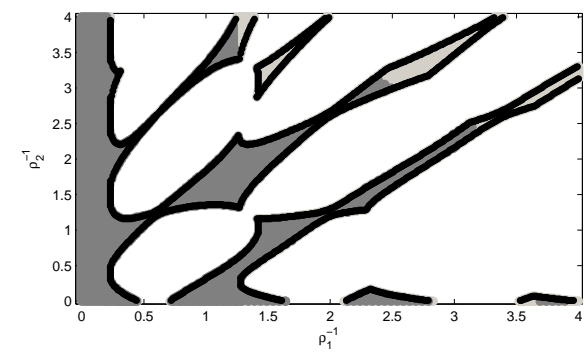

(b) Figure representing the unstable region (white area), the stability region guaranteed by Theorem 1 with $\delta=0$ and $N=10$ (grey) and the region of stability for which no solution has been obtained with the same $N=10$ (light grey).

Figure 2: Stability regions in the plane $\left(\rho_{1}^{-1}, \rho_{2}^{-1}\right)$ for Examples 3 and 4.

It is also important to note that Theorem 1 does not rely on the application of the Lyapunov-Krasovskii theorem as usual for time-delay systems. Theorem 1 is based on the application of Lyapunov theorem for infinite dimensional systems. However, when the coupled system (1) can be interpreted as a time-delay system, one can easily prove that its energy $E(t)$ verifies $|X(t)|_{n}^{2} \leq E(t) \leq \eta \sup _{s \in[-h, 0]}|X(s)|_{n}^{2}$, for all $t>0$, where $h=\max _{i=1, \ldots, p}\left(\rho_{i}^{-1}\right)$, inequalities (16) also implying

$$
\begin{aligned}
& \varepsilon_{1}|X(t)|_{n}^{2} \leq V(X, z) \leq \varepsilon_{2} \eta \sup _{s \in[-h, 0]}|X(s)|_{n}^{2}, \\
& \dot{V}(X, z)+2 \delta V(X, z) \leq-\varepsilon_{3}|X(t)|_{n}^{2} .
\end{aligned}
$$

which ensures the stability of system (1) in the sense of the LyapunovKrasovskii Theorem. This shows that this formulation does not bring any restriction with the usual Lyapunov-Krasovskii Theorem.

\subsection{Example 4}

Finally, let us consider system (1) with the matrices $A=\left[\begin{array}{cc}0 & 1 \\ -20 & -1\end{array}\right]$,

$$
B=\left[\begin{array}{cccccc}
0 & 0 & 0 & 0 & 0 & 0 \\
-3 & -2 & -4 & -1 & -1 & 0
\end{array}\right], C_{1}=\left[\begin{array}{l}
I_{2} \\
I_{2} \\
0_{2}
\end{array}\right], C_{2}=\left[\begin{array}{ccc}
0_{2} & 0_{2} & 0_{2} \\
0_{2} & 0_{2} & 0_{2} \\
I_{2} & 0_{2} & 0_{2}
\end{array}\right], \Lambda=\left[\begin{array}{cc}
\rho_{1} I_{2} & 0 \\
0 & \rho_{2} I_{4}
\end{array}\right]
$$

Figure 2(b) depicts the stability regions obtained by the conditions of Theorem 1 in the plane $\left(\rho_{1}^{-1}, \rho_{2}^{-1}\right)$ for $N=10$. Using a similar argument, this 
particular example can be rewritten as a linear systems with commensurate delays, allowing us to draw the exact stability regions based on a frequency approach (for instance the one exploited in [32]. One can check that Theorem 1 is able to provide a very good inner approximation of the stability region, matching with the region obtained through the frequency domain analysis (black boundaries in Figure 2(b)).

A final comment dedicated to the time-delay systems analysis deals with the fact that usual Lyapunov-Krasovskii analysis of such systems with commensurate delays would introduce three independent delays, i.e. $\rho_{1}^{-1}, \rho_{2}^{-1}$ and also $\rho_{1}^{-1}+\rho_{2}^{-1}$, which misses the link between these three delays. The methodology provided in our present article allows to deal with this class of systems in a direct and generic manner.

\section{Conclusion}

In this article we give a general presentation of a system coupling ODEs with a vectorial transport PDE, and we provide a new approach for the stability analysis of this kind of systems. The approach consists in a Lyapunov method that gives LMI conditions depending on the transport speed matrix, on the degree of the approximation which is based on Legendre polynomials and on the guaranteed decay rate $\delta$ of the energy of the system. This work gives a more general setting for the analysis than the one provided in [3] which takes into account a single transport speed and no cross talking transport states. This paper also generalizes the work proposed in [31], on single constant transport speed, to the case of multiple speeds. In addition, we prove that the set of stability conditions forms a hierarchy of LMI indexed by the polynomial degree $N$, in the sense that increasing $N$ reduces the conservatism of the proposed method.

This stability study can be seen as a milestone for future research on infinite dimensional systems. A first direction of research would be to extend this stability study to uncertain or time-varying speed matrices $\Lambda$. We also aim at extending such an analysis to systems coupling ODEs with a wider class of PDEs, including for instance heat equation or wave equation among many others.

\section{References}

[1] M. Ahmadi, G. Valmorbida, and A. Papachristodoulou. Input-output analysis of distributed parameter systems using convex optimization. In 53rd IEEE Conference on Decision and Control, pages 4310-4315, Dec 2014.

[2] G. Bastin and J-M. Coron. Stability and boundary stabilization of 1-d hyperbolic systems. Springer, 2016. 
[3] L. Baudouin, A. Seuret, and M. Safi. Stability analysis of a system coupled to a transport equation using integral inequalities. IFAC Conference on Control of Systems Governed by Partial Differential Equations, Bertinoro, Italy, 2016.

[4] N. Bekiaris-Liberis and M. Krstic. Nonlinear control under nonconstant delays, volume 25 of Advances in Design and Control. Society for Industrial and Applied Mathematics (SIAM), Philadelphia, PA, 2013.

[5] F. Castillo, E. Witrant, and L. Dugard. Contrôle de température dans un flux de poiseuille. In IEEE Conférence Internationale Francophone d'Automatique, Grenoble, France, 2012.

[6] F. Castillo, E. Witrant, C. Prieur, and L. Dugard. Dynamic boundary stabilization of first order hyperbolic systems. In Recent Results on Time-Delay Systems, pages 169-190. 2016.

[7] W-H. Chen, Z-H. Guan, and X. Lu. Delay-dependent exponential stability of uncertain stochastic systems with multiple delays: an LMI approach. Systems 85 Control Letters, 54(6):547 - 555, 2005.

[8] R-M. Colombo, G. Guerra, M. Herty, and V. Schleper. Optimal control in networks of pipes and canals. SIAM Journal on Control and Optimization, 48(3):2032-2050, 2009.

[9] J-M. Coron. Control and nonlinearity, volume 136 of Mathematical Surveys and Monographs. American Mathematical Society, Providence, RI, 2007.

[10] R. Courant and D. Hilbert. Methods of mathematical physics. Interscience publishers, New York, 1, 1953.

[11] S. Damak, M. Di Loreto, and S. Mondié. Stability of linear continuous-time difference equations with distributed delay: Constructive exponential estimates. International Journal of Robust and Nonlinear Control, 25(17):31953209, 2015.

[12] F. Di Meglio, R. Vazquez, and M. Krstic. Stabilization of a system of $n+1$ coupled first-order hyperbolic linear PDEs with a single boundary input. IEEE Transactions on Automatic Control, 58(12):3097-3111, 2013.

[13] M. Dick, M. Gugat, and G. Leugering. Classical solutions and feedback stabilization for the gas flow in a sequence of pipes. NHM, 5(4):691-709, 2010.

[14] A. Egorov and S. Mondié. Necessary stability conditions for linear delay systems. Automatica, 50(12):3204-3208, 2014.

[15] E. Fridman. Introduction to time-delay systems. Systems \& Control: Foundations \& Applications. Birkhäuser/Springer, Cham, 2014. Analysis and control. 
[16] K. Gu, J. Chen, and V. L. Kharitonov. Stability of time-delay systems. Springer Science \& Business Media, 2003.

[17] E. Gyurkovics and T. Takacs. Multiple integral inequalities and stability analysis of time delay systems. Systems \& Control Letters, 96:72 - 80, 2016.

[18] L. Hu, F. Di Meglio, R. Vazquez, and M. Krstic. Control of homodirectional and general heterodirectional linear coupled hyperbolic pdes. IEEE Transactions on Automatic Control, 61(11):3301-3314, 2016.

[19] E. Jarlebring. Computing critical delays for time delay systems with multiple delays. Proc. Reglermötet, Stockholm, Sweden, 2006.

[20] M Krstic. Delay compensation for nonlinear, adaptive, and PDE systems. Springer, 2009.

[21] M. Krstic and A. Smyshlyaev. Backstepping boundary control for first-order hyperbolic pdes and application to systems with actuator and sensor delays. Systems 83 Control Letters, 57(9):750-758, 2008.

[22] Z.-H. Luo, B.-Z. Guo, and O. Morgul. Stability and stabilization of infinite dimensional systems with applications. Communications and Control Engineering Series. Springer-Verlag London, Ltd., London, 1999.

[23] S. Mondie, V. L. Kharitonov, and O. Santos. Complete type lyapunovkrasovskii functionals with a given cross term in the time derivative. In Proceedings of the 44th IEEE Conference on Decision and Control, pages 5060-5064, Dec 2005.

[24] S-I Niculescu. Delay effects on stability: a robust control approach, volume 269. Springer Science \& Business Media, 2001.

[25] N. Olgac, T. Vyhlídal, and R. Sipahi. Exact stability analysis of neutral systems with cross-talking delays. In Time Delay Systems, volume 6, pages 175-180, 2006.

[26] A. Papachristodoulou and M.M. Peet. On the analysis of systems described by classes of partial differential equations. In Proc. of the 45th IEEE Conf. on Decision and Control, San Diego, CA, USA, pages 747-752, 2006.

[27] M. M. Peet, A. Papachristodoulou, and S. Lall. Positive forms and stability of linear time-delay systems. SIAM Journal on Control and Optimization, 47(6):3237-3258, 2009.

[28] C. Prieur, J. Winkin, and G. Bastin. Robust boundary control of systems of conservation laws. Mathematics of Control, Signals, and Systems, 20(2):173197, 2008. 
[29] M. Safi, L. Baudouin, and A. Seuret. Refined exponential stability analysis of a coupled system. In IFAC World Congress, Toulouse, France, July 2017.

[30] A. Seuret and F. Gouaisbaut. Wirtinger-based integral inequality: application to time-delay systems. Automatica, 49(9):2860-2866, 2013.

[31] A. Seuret and F. Gouaisbaut. Hierarchy of LMI conditions for the stability analysis of time-delay systems. Systems $\&$ Control Letters, 81:1-7, 2015.

[32] R. Sipahi, S-I. Niculescu, C. T. Abdallah, W. Michiels, and K. Gu. Stability and stabilization of systems with time delay. IEEE Control Systems, 31(1):3865, Feb 2011.

[33] J-H Su. The asymptotic stability of linear autonomous systems with commensurate time delays. IEEE Transactions on Automatic Control, 40(6):11141117, Jun 1995.

[34] G-A. Susto and M. Krstic. Control of PDE-ODE cascades with neumann interconnections. Journal of the Franklin Institute, 347(1):284 - 314, 2010. Dynamics and Control.

[35] Y. Tang, C. Prieur, and A. Girard. Stability analysis of a singularly perturbed coupled ODE-PDE system. In 2015 54th IEEE Conference on Decision and Control (CDC), pages 4591-4596, Dec 2015.

[36] M. Zainea, A. Van Der Schaft, and J. Buisson. Stabilizing control for power converters connected to transmission lines. In 2007 American Control Conference, pages 3476-3481, New-York city, United States, July 2007. 